\title{
Medicamentos disponibles en España para la pérdida de peso previo a la artroplastia total de articulaciones.
}

DOI: http//dx.doi.org/10.37315/SOTOCAV20212865627

NOVOA-PARRA CD 1, MODREGO-PARDO I 2, MORILLAS-ARIÑO C 2,3.

1 SERVICIO DE CIRUGÍA ORTOPÉDICA Y TRAUMATOLOGÍA. HOSPITAL DE DENIA, ALICANTE, ESPAÑA

2 SERVICIO DE ENDOCRINOLOGÍA Y NUTRICIÓN. HOSPITAL MARINA BAIXA DE LA VILA JOIOSA. ALICANTE, ESPAÑA

3 UNIVERSIDAD DE VALENCIA. VALENCIA, ESPAÑA.

\section{CARTA AL DIRECTOR.}

Como usted bien sabe la obesidad es un problema de salud actual. Según la Encuesta Nacional de Salud en 2017 un $17,43 \%$ de los españoles la padecen. Además, en nuestras consultas es un problema recurrente los pacientes que presentan una artropatía y obesidad concomitante. Actualmente, existen medicamentos seguros y eficaces para la pérdida de peso, que nosotros como cirujanos ortopédicos, con un conocimiento adecuado, podemos prescribir en busca de mejorar la salud de los pacientes y los resultados quirúrgicos. En este pequeño resumen intentamos llevar este conocimiento a los especialistas de nuestro ámbito.

\section{LETTER TO THE EDITOR.}

As you well know, obesity is a current health problem. According to the National Health Survey in 2017, 17.43\% of Spaniards suffer from it. Furthermore, in our consultations, patients with concomitant arthropathy and obesity are a recurring problem. Currently, there are safe and effective medications for weight loss, which we as orthopedic surgeons, with adequate knowledge, can prescribe in search of improving the health of patients and surgical results. In this short summary we try to bring this knowledge to specialists in our field.

\author{
Correspondencia: \\ Carlos Daniel Novoa Parra \\ Telf.: 697730175 \\ e-mail: le_male2002@hotmail.com \\ Dirección postal: Av. Marina Alta, s/n, 03700 Dénia, Alacant.
}




\section{MEDICAMENTOS DISPONIBLES EN ESPAÑA PARA LA PÉRDIDA DE PESO PREVIO A LA ARTROPLASTIA TOTAL DE ARTICULACIONES (Carta).}

Históricamente, se pensaba que la obesidad promovía la artrosis a través de un mecanismo de desgaste; sin embargo, la evidencia reciente ha apuntado a la influencia hormonal de la obesidad como un factor importante en su desarrollo. La teoría biomecánica de que la obesidad conduce a un aumento de las fuerzas articulares y al desgaste del cartílago tiene poco soporte científico ${ }^{1}$. Ahora se teoriza que el estado inflamatorio sistémico de bajo grado creado por el exceso de función de los adipocitos causa efectos patológicos directos sobre el sistema musculoesquelético. A nivel celular, la inflamación creada por el aumento de tejido adiposo conduce a estrés oxidativo y endotelial ${ }^{1}$. Más específicamente, las citocinas proinflamatorias liberadas desde el tejido adiposo interactúan con componentes del sistema musculoesquelético como cartílago, hueso, tejido meniscal y células sinoviales, lo que resulta en degradación ósea y subcondral, así como inflamación de tejidos blandos ${ }^{1}$.

Por lo tanto, la obesidad es un factor de riesgo independiente para el desarrollo de artrosis, y los pacientes con exceso de adiposidad se someten a una artroplastia a una edad más temprana y a su vez la artroplastia en esta población de pacientes tiene un riesgo elevado de infección, reoperación, inestabilidad y procedimientos quirúrgicos de revisión ${ }^{2}$. Debido a que el peso preoperatorio puede reducir la tasa de complicaciones asociadas con la cirugía protésica, optimizarlo antes de la artroplastia debe ser una preocupación primordial para los cirujanos ortopédicos. Este desafío aumenta cuando fallan las medidas conservadoras para la reducción de peso, como las modificaciones en el estilo de vida en forma de dieta y ejercicio. En este contexto, la cirugía o la medicación podrían ser una opción.

La cirugía bariátrica se ha utilizado antes de la artroplastia para reducir el peso preoperatorio; sin embargo, una revisión sistemática demostró que la evidencia sobre la eficacia de la cirugía bariátrica, antes de la cirugía protésica, permanece en entredicho sobre si se observan reducciones de las complicaciones posoperatorias $^{3}$.

El uso de medicamentos contra la obesidad no es común en nuestra práctica clínica, posiblemente debido a las preocupaciones de los cirujanos ortopédicos con respecto a su uso y la exposición insuficiente a las evidencias preexistentes. Actualmente, existen 3 medicamentos contra la obesidad aprobados por la AEMPS (Agencia Española de Medicamentos y Productos Sanitarios): Orlistat, Naltrexona/bupropion de liberación prolongada y Liraglutida. Estos brindan potencialmente una nueva forma de optimizar a los pacientes antes de la cirugía y de garantizar una recuperación postoperatoria exitosa.

En cuanto al mecanismo de acción ${ }^{2}$, el Orlistat actúa inhibiendo las lipasas gastrointestinales, evitando la absorción de aproximadamente el $30 \%$ de las grasas de la dieta. Respecto a la Naltrexona/bupropion, el compuesto naltrexona actúa inhibiendo la autoinhibición de proopiomelanocortina mediada por receptores opioides, y el bupropion inhibe selectivamente la recaptación de dopamina y noradrenalina. Trabajando de manera sinérgica, estos medicamentos son efectivos para reducir la ingesta acumulada de alimentos y aumentar la saciedad. Estos medicamentos tienen un efecto sobre las vías de recompensa del sistema nervioso central, la ingesta de alimentos y la saciedad, a través de la inhibición por retroalimentación antagonista y se han utilizado individualmente para tratar la adicción. La Liragutida, es un análogo del péptido 1 similar al glucagón humano (GLP-1) con amplia experiencia en su uso como tratamiento de la diabetes mellitus tipo 2 ya que estimula la secreción de insulina de un modo dependiente de la glucosa (hipoglicemiante). En cuanto a los mecanismos por los cuales favorece la pérdida de peso se trata de una combinación de efectos a nivel del tracto gastrointestinal (retrasando el vaciado gástrico) y a nivel central favoreciendo la reducción del apetito y de la ingesta calórica.

Como en toda intervención médica la valoración del riesgo beneficio es crucial. En un metaanálisis de ensayos clínicos aleatorizados $(E C A)^{4}$ todos los fármacos analizados en nuestro estudio se asociaron con pérdida de peso significativa frente a placebo al año: Orlistat, -2,6 kg (IC95\% $-3,04$ a $-2,16 \mathrm{~kg})$; Naltrexona/bupropion, $-5,0 \mathrm{~kg}$ (IC95\% $-5,94$ a $-3,96 \mathrm{~kg}$ ); y Liraglutida, $-5,3 \mathrm{~kg}$ (IC95\% -6,06 a -4,52 kg). De igual manera se asociaron con una mayor proporción de pacientes que hayan alcanzado una pérdida de peso $\geq 5 \%$ frente a placebo: Orlistat (59\% de los pacientes en tratamiento) con una odds ratio (OR) de 2,69 (IC95\%, 2,36-3.07), Naltrexona/bupropion (53\%) OR de 3,90 (IC95\% 2.91-5.22), y Liraglutida $(61,5 \%)$ con un OR de 5,09 (IC95\% 4,07-6.37); y con una mayor proporción de pacientes que hayan alcanzado una pérdida de peso $\geq 10 \%$ frente a placebo: Orlistat (31,3\% de los pacientes tratados) OR de 2,41 (IC95\% 2,082.78 ), Naltrexona/bupropion (29,3\%) OR de 4.11 (IC95\% 2.806.05), y Liraglutida (32,8\%) con un OR de 4.36 (IC95\% 3.61-5.26). Además, comparativamente con el Orlistat la Liraglutida se asoció con una mayor proporción de pacientes que habían alcanzado una pérdida de peso $\geq 5 \%$ OR 3.66 (IC95\% 1.79-7.46); y con una mayor proporción de pacientes que habían alcanzado una pérdida de peso $\geq 10 \%$ OR 3.87 (IC95\% 1.65-9.04).

En cuanto a los efectos adversos, contraindicaciones y advertencias $^{2}$, debido a su mecanismo de acción, se informó que los efectos adversos del Orlistat incluyen malabsorción, diarrea, heces grasas y dolor abdominal, y está contraindicado en pacientes con síndrome de malabsorción o colestasis. Así mismo, y en especial en los pacientes añosos manejados en nuestra especialidad, los pacientes que actualmente están tomando anticoagulantes antagonistas de la vitamina $\mathrm{K}$ deben tener sus parámetros hemostáticos monitoreados de cerca, ya que una disminución potencial en la absorción de vitamina $K(A V K)$ puede justificar una reducción de la dosis prescrita de AVK. El Orlistat también puede inhibir la absorción de amiodarona y ciclosporina, y requiere una vigilancia estrecha de los niveles de medicación antiepiléptica ${ }^{2}$.

La Naltrexona/bupropion, como efectos adversos más destacables presenta cefalea, sequedad de boca, mareos, náuseas, vómitos y estreñimiento. La Naltrexona/bupropion está contraindicada en pacientes con antecedentes de convulsiones, adicción a las drogas, uso de opioides y bulimia y / o anorexia nerviosa, que estén recibiendo cualquier tratamiento simultáneo que contenga bupropion o naltrexona, o que estén recibiendo inhibidores de la monoaminoxidasa (IMAO) $)^{2}$.

Los eventos adversos notificados con mayor frecuencia con Liraglutida son náuseas y diarrea, aunque fueron raros y de naturaleza leve. La Liraglutida está contraindicada en pacientes con antecedentes de carcinoma medular de tiroides y no esta indicada en diabetes tipo $\mathrm{I}^{2}$.

Los efectos adversos previamente descritos pueden llevar al abandono de la medicación ${ }^{4}$. El fármaco que mayor asociación tuvo entre los efectos secundarios y el abandono de la medicación fue la Liraglutida OR 2.82 (IC95\% 2.10-3.77) comparada con placebo, y cuando se comparaba con el Orlistat este riesgo aumenta a OR 3.50 (IC95\% 0.70-17.49). Comparadas con placebo, la que menor tasa de abandonos presentaba era el Orlistat OR 1.84 (1.55-2.18), seguido por la Naltrexona/bupropion OR 2.60 (2.15-3.14). Sin embargo, comparado con placebo, cuando se observan los intervalos de confianza no es posible establecer diferencias significativas entre estas medicaciones en cuanto al riesgo de abandono. 
En la tabla 1 se realiza un resumen de las indicaciones, posología y recomendaciones para el uso de los medicamentos contra la obesidad aprobados en España. El tratamiento con Liraglutida debe ser considerado la primera opción entre las tres opciones farmacológicas actualmente disponibles en Europa siguiendo la recomendación de la Sociedad Española para el Estudio de la Obesidad (SEEDO) ${ }^{5}$ debido a la amplia experiencia de uso disponible, a la mayor potencia obtenida en la reducción ponderal, a los escasos y conocidos efectos adversos y a los demostrados efectos beneficiosos sobre la enfermedad cardiovascular. Es importante recalcar que los estudios en los que se basan estas recomendaciones ${ }^{2,3}$ no encontraron ECA de pacientes sometidos a artroplastia articular total, sino en la población obesa en general. Sin embargo, estos son análisis de ensayos clínicos y la evidencia más poderosa que evalúa el uso de estos medicamentos para la reducción de peso que, como hemos dicho, nos brindan una nueva forma de optimizar a nuestros pacientes antes de un procedimiento quirúrgico y de asegurar una recuperación exitosa después del mismo.

Tabla 1: Medicación aprobadaen España, indicaciones, posologia y racomendaciones bas adaen las fichas técnicas AEMPS-CTMA (centro de información online demedicamentos autorizados) y el estudio de Amanatullah et al [2]

\begin{tabular}{|c|c|c|c|}
\hline Medicación & Orlistat & Naltrexona/Bupropion de liberación sostenida & Liraglutidat+t \\
\hline Indicación & \multicolumn{3}{|c|}{ MCC $\geq 30 \mathrm{~kg} / \mathrm{m}_{2} \circ 27 \mathrm{~kg} / \mathrm{m} 2$ con factores de tieago cardiovacular } \\
\hline $\begin{array}{l}\text { Financiado por SNS para } \\
\text { obetidad }\end{array}$ & No & No & No \\
\hline $\begin{array}{l}\text { Via de administracióny } \\
\text { dosis }\end{array}$ & 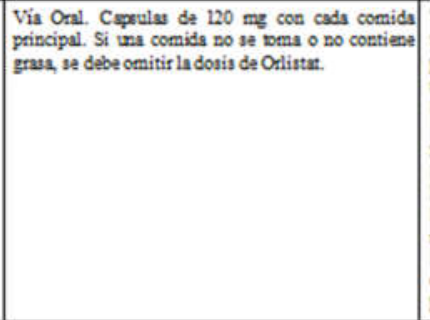 & 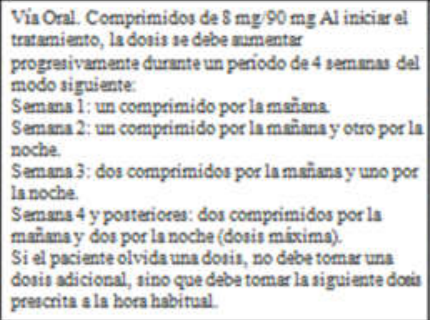 & 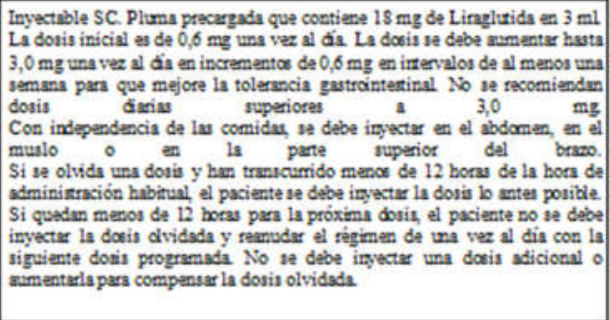 \\
\hline $\begin{array}{l}\text { Duración (en assencia da } \\
\text { efectos adversos sin } \\
\text { limite en todos los casos, } \\
\text { excepto) }\end{array}$ & $\begin{array}{l}\text { Si tras } 16 \text { semanas los pacientes no han perdido al } \\
\text { menos el } 5 \% \text { de rupeno corporal inicial suspender }\end{array}$ & $\begin{array}{l}\text { Si tras } 12 \text { semmas los pacientes no han perdido al } \\
\text { menos el } 5 \% \text { de us paso conporal inicial surpender }\end{array}$ & $\begin{array}{l}\text { Si tras } 12 \text { semanas los pacientes no han perdido al menos el } 59 \text { de su paso } \\
\text { comporal inicial suspender }\end{array}$ \\
\hline Edad $65-75$ a alos & No eatudiado & Precsución (posible mumento de los efactos nerviosos) & Utilizar \\
\hline Edad $\geq 75$ años & No entudiado & No recomendado & No recomendado \\
\hline Hipertensión controlads & Utilizer & $\begin{array}{l}\text { Precusción (control periódico de cifas de tensión } \\
\text { anterial) }\end{array}$ & Utilizal \\
\hline $\begin{array}{l}\text { Hipenensión no } \\
\text { controlada }\end{array}$ & Utilizar & Eviter & Utiliza \\
\hline Disbotes tipo II & \multirow[t]{2}{*}{ Precaución (monitorizar tratamiento sntidiabético) } & Utilizar & Utilizar (preferible) \\
\hline Predisbetes & & Utilizar & Utilizar (preferible) \\
\hline Enfemedad coronaria & Utilizar & Precrución & Utilizar (preferible) \\
\hline
\end{tabular}

\begin{tabular}{|c|c|c|c|}
\hline Astitmia & $\begin{array}{l}\text { Precución. (diminución de la sbuotción de } \\
\text { amioderona) }\end{array}$ & Precsución & Utilizar \\
\hline $\begin{array}{l}\text { Madicación } \\
\text { anticosgulante }\end{array}$ & Precusción. (disminución de la absorción de AVK) & Utilizar & Pracusción (bematomas en sitio del pinchazo) \\
\hline $\begin{array}{l}\text { Historial de convulasiones } \\
\text { oepilepuis }\end{array}$ & $\begin{array}{l}\text { Precusción (diminución de la sboorción de } \\
\text { medicasión antiepiléptica) }\end{array}$ & Evitar & Utilizar \\
\hline $\begin{array}{l}\text { Enfemedad renal crónica } \\
\text { (levermoderada) }\end{array}$ & \multirow{3}{*}{ 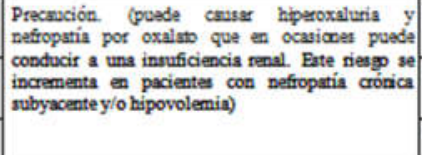 } & Precaución (valorar disminuir dosis) & Utilizar \\
\hline $\begin{array}{l}\text { Enfermadad renal crónics } \\
\text { (grwe) }\end{array}$ & & Eviter & Precaución \\
\hline Cáloulos reneles & & Utiliza & Utilizar \\
\hline $\begin{array}{l}\text { Enfermedad bepitica } \\
\text { (leve-moderada) }\end{array}$ & Evitar & Evitar & Utilizar \\
\hline $\begin{array}{l}\text { Enfermedad bepitica } \\
\text { (severs) }\end{array}$ & Evitar & Evitur & Evitar \\
\hline Uso de opioides & Utilizar & Eviter & Utilizar \\
\hline Glencoma & Utilizar & Evitar & Utilizar \\
\hline Pencrestitis & Precusción & Utilizar & Evitar \\
\hline Depreatión & Utilizar & \multirow{2}{*}{$\begin{array}{l}\text { Utilizar (preferible). Sin mbargo, en nacesario } \\
\text { controlar la aperición de alteraciones del entado de } \\
\text { inimo al utilizar eata medicación }\end{array}$} & Utilizar \\
\hline Ansiedad & Utilizar & & Utiliza \\
\hline Trastomo por atracón & 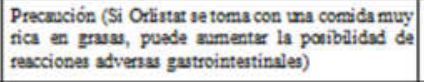 & Utilizar (preferible) & Utilizar \\
\hline Embaraso $\circ$ lactancia & Eviter & Evitar & Evitar \\
\hline $\begin{array}{l}\text { Eficto sobre la mentesis } \\
\text { perioperatoris }\end{array}$ & No conocido & $\begin{array}{l}\text { No conocido Se recomienda no tomar en las } 24 \text { horas } \\
\text { previas a la intervención pers evitar el efacto } \\
\text { inhibitorio sobre la scción analgéaica de los opioiden }\end{array}$ & $\begin{array}{l}\text { No conocida. Al enlentecer el vaciemient girtrico hay que comiderat } \\
\text { prologar el ayuno preoperatotio }\end{array}$ \\
\hline
\end{tabular}

La información contenida en esta tabla debe interpretarse como una guia para el uso de la medicación que no sustuye al conocimiento y criterio del facultativo prescriptor. Se debe aconsejar a los pacientes que sigan las recomendaciones dietéticas que les sean dadas. "**(Saxenda\& es el nombre comercial del medicamento con indicación en obesidad, Victoza solo esta indicado en pacientes con Obesidad y diabetes tipo II concomitante. No estan indicados en diabetes tipo I). 


\section{Bibliografía}

1. Koonce RC, Bravman JT. Obesity and osteoarthritis: more than just wear and tear. J Am Acad Orthop Surg. 2013; 21(3):161-169. doi:10.5435/JAAOS-21-03-161

2. Amanatullah, DF, Ohanisian, L, Bailony R. Medications available for weight reduction in elective total joint arthroplasty. 2020; 8(6):1-11. doi: 10.2106/JBJS.RVW.19.00123

3. Gu A, Cohen JS, Malahias MA, Lee D, Sculco PK, McLawhorn AS. The effect of bariatric surgery prior to lower-extremity total joint arthroplasty: A Systematic Review. HSS J. 2019; 15(2):190-200. doi:10.1007/s11420-019-09674-2

4. Khera R, Murad MH, Chandar AK, Dulai, PS, Wang Z, Prokop LJ. Association of Pharmacological Treatments for Obesity With Weight Loss and Adverse Events: A Systematic Review and Meta-analysis. JAMA. 2016; 315(22):2424-2434. https://doi.org/10.1001/jama.2016.7602

5. Lecube A, Freitas P, Monereo S, Souto S, Tinahones F. Consenso ibérico sobre el tratamiento farmacológico de la obesidad en el paciente adulto: Posicionamiento de las Sociedades Española y Portuguesa para el Estudio de la Obesidad (SEEDO-SPEO) 2018. Bariatr Metab Iberoam. 2018; 8:2325-43.35. 